\title{
Outcomes of vitrectomy for advanced diabetic retinopathy at Groote Schuur Hospital, Cape Town, South Africa
}

\author{
J C Rice, MB BCh, MRCOphth, FCOphth (SA), MPH (Clinical Research); J Steffen, MB ChB, FCOphth (SA) \\ Division of Ophthalmology, Faculty of Health Sciences, University of Cape Town and Groote Schuur Hospital, Cape Town, South Africa
}

Corresponding author: J C Rice (james.rice@uct.ac.za)

\begin{abstract}
Background. Present limitations in primary and secondary prevention of diabetic retinopathy mean that many patients with diabetes present with advanced retinal complications, often requiring surgery (vitrectomy).

Objectives. To determine the outcomes of vitrectomy for advanced diabetic retinopathy and to examine context-specific risk factors that may influence outcomes and decisions affecting resource allocation.

Methods. This was a retrospective cohort study of 124 vitrectomies with up to 6 months' follow-up.

Results. Visual acuity was $6 / 60$ or worse in the better eye in $23.4 \%$ of patients at presentation. The mean visual acuity of the listed eye was $2 / 60$. The fellow eye was considered inoperable in $20.2 \%$ of cases. Visual function declined significantly in $26.2 \%$ of patients while awaiting surgery. The average waiting time until surgery was 2.9 months (range 1 day - 9 months). Epiretinal membranes were present in $93.6 \%$ of cases, and posterior iatrogenic breaks occurred in $49.2 \%$. Silicone oil was used in $24.2 \%$. Visual acuity improved in $54.9 \%$, was unchanged in $30.1 \%$, and worsened in $14.0 \%$ of cases at 6 months. Patients with poorer vision at surgery were more likely to improve (odds ratio 2.15 ; $p=0.048)$. Factors associated with a worse visual outcome were increased age at surgery $(p=0.042)$ and posterior iatrogenic retinal breaks $(p=0.007)$. Renal dysfunction was not associated with worse visual outcomes.

Conclusion. Vitrectomy improved or stabilised vision in $85.0 \%$ of cases, although outcomes were unpredictable. A long waiting time to surgery contributed to patient morbidity. The presence of renal dysfunction did not predict poorer visual outcomes.

S Afr Med J 2015;105(6):496-499. DOI:10.7196/SAMJ.9203
\end{abstract}

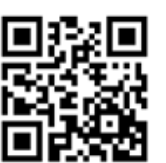

Diabetic blindness is a preventable condition. Unfortunately diabetic screening and early intervention are not yet available for most of the diabetic population in the state sector in South Africa (SA). A recent review of 248 diabetic patients attending a day hospital in Cape Town revealed that only $5.2 \%$ had regular annual fundus examinations and only $10.4 \%$ were aware that annual fundoscopy was required. ${ }^{[1]}$ Although screening programmes have been piloted or partially implemented in a few localised centres, ${ }^{[2,3]}$ the severe deficiencies in current screening and laser management mean that large numbers of patients present with advanced ocular complications of diabetes. Management of these patients usually requires surgical intervention (vitrectomy), and visual outcomes remain unpredictable.

Fifty per cent of vitrectomies performed at Groote Schuur Hospital (GSH), Cape Town, are for the complications of diabetic retinopathy, and we anticipate that the proportion may be similar at other state hospitals. In resource-limited settings it is appropriate to examine the outcomes of retinal surgery and to assess context-specific risk factors that may influence surgical outcomes, as well as decisions affecting resource allocation. We are not aware of any publications of this type from SA state facilities.

\section{Methods}

We performed a retrospective cohort study of consecutive patients undergoing diabetic vitrectomy at GSH from January 2012 to December 2012 with up to 6 months' follow-up. All patients were assessed and listed for surgery by four vitreoretinal consultants (one full-time and three sessional consultants) and one trainee vitreoretinal consultant in the department. Preoperative visual acuities were recorded using Snellen charts. The time from listing for surgery to the date of the operation was recorded, as well as any change in visual acuity during this period. Surgical cancellations or postponements were recorded. Routine preoperative tests included an assay for urea and electrolytes.

Intraoperative details were recorded by each surgeon. Early (within 1 month) and late postoperative complications were noted. If silicone oil was inserted during the procedure, time until removal of oil was recorded. Postoperative visual acuity was assessed at 3 and 6 months, and cases were considered anatomical successes if the retina was completely attached with no features of proliferation in the absence of a tamponading agent (e.g. silicone oil).

All data were collected in Epidata and transferred to STATA 12 (Stata Statistical Software: Release 12, StataCorp, USA) for analysis. Visual acuity was converted to the logarithm of the minimum angle of resolution (LogMAR) for statistical analysis. The $\chi^{2}$ test or Fisher's exact test was used for categorical variables and Student's $t$-test and the rank sum test for continuous variables. Logistic regression was used to examine prognostic factors.

The study was approved by the University of Cape Town Human Research Ethics Committee (approval number: HREC 851/2014).

\section{Results}

During the study period, 124 vitrectomies were performed on 115 patients, 18 (15.6\%) of whom were from rural areas. Seventyone $(61.7 \%)$ were female. The mean age at surgery was 57 years. Indications for surgery are listed in Table 1.

Visual acuity was LogMAR 1.0 (Snellen 6/60) or worse in the better eye in $23.4 \%$ of patients at presentation. At the time of listing for surgery, the mean visual acuity of the involved eye was LogMAR 1.45 (Snellen $2 / 60$ ). Sixty eyes (48.8\%) were classified as counting fingers or worse. Three patients had perception of light only (Table 2). The patient's fellow eye was considered inoperable in $20.2 \%$ of cases.

The average waiting time until surgery was 2.9 months (range 1 day - 9 months) (Fig. 1). During this time $26.2 \%$ of eyes experienced 
Table 1. Indications for surgery

\begin{tabular}{ll}
\hline Indication & $\boldsymbol{n}(\mathbf{\%})$ \\
\hline Non-clearing vitreous haemorrhage & $21(16.9)$ \\
Tractional retinal detachment affecting the macula & $37(29.8)$ \\
CTR & $20(16.1)$ \\
Macular traction and vitreous haemorrhage & $43(34.7)$ \\
Other & $3(2.4)$ \\
Total & 124 \\
CTR = combined tractional rhegmatogenous retinal detachment. &
\end{tabular}

Table 2. Presenting visual acuity of the operated eye

\begin{tabular}{lll}
\hline LogMAR acuity & Snellen equivalent & $\boldsymbol{n}(\%)$ \\
\hline 0.18 & $6 / 9$ & $4(3.3)$ \\
0.3 & $6 / 12$ & $4(3.3)$ \\
0.48 & $6 / 18$ & $19(15.5)$ \\
0.6 & $6 / 24$ & $9(7.3)$ \\
0.78 & $6 / 36$ & $8(6.5)$ \\
1.0 & $6 / 60$ & $18(14.6)$ \\
1.3 & $3 / 60$ & $1(0.8)$ \\
2.0 & $\mathrm{CF}$ & $39(31.7)$ \\
3.0 & $\mathrm{HM}$ & $18(14.6)$ \\
5.0 & $\mathrm{PL}$ & $3(2.4)$ \\
Total & & $123(100.0)$ \\
CF = count fingers; HM = hand motions; PL = perception of light. &
\end{tabular}

a worsening of visual acuity, dropping from a mean of LogMAR 0.84 to LogMAR 1.84 (Snellen $6 / 40$ to $<1 / 60 ; p<0.0001$ ). One patient dropped from $6 / 24$ to perception of light only (Fig. 2).

Surgery was cancelled or postponed at least once in 15 cases (12.1\%). The most common reasons for cancellation were shortage of surgery time on the day $(n=7)$ and poor systemic health with high anaesthetic risk $(n=6)$. In 54 cases $(43.5 \%)$ estimated glomerular filtration rates revealed moderate renal dysfunction or worse at the time of surgery; nine patients had severe renal dysfunction and two renal failure.

All surgeries were performed using the Constellation Vision System (Alcon Laboratories). Surgery was performed by one full-time consultant, three part-time consultants and one trainee vitreoretinal consultant. Fifty-one per cent of procedures were performed using 23-gauge, $48.4 \%$ using 20 -gauge and $0.8 \%$ using 25 -gauge instrumentation. Cataract surgery was performed at the same time in $54.0 \%$ of cases. Epiretinal membranes were present in $93.6 \%$ of patients and any degree of macular traction in $79.0 \%$. Posterior iatrogenic retinal breaks occurred in $49.2 \%$ of cases and port-related tears in $7.8 \%$.

To achieve intraocular tamponade, silicone oil was used in $24.2 \%$ of cases, SF6 gas in $23.4 \%$, C3F8 gas in $7.3 \%$, and air in $7.3 \%$; in $37.9 \%$ no tamponade was necessary. Of the patients in whom silicone oil was used (30 cases), 6 (20.0\%) had significant haemorrhage under the oil in the early postoperative period. The mean duration of time until the silicone oil was removed was 9.9 months (range 5.3 - 16.8).

Raised intraocular pressure $(>21 \mathrm{mmHg}$ ) was the most common early postoperative complication (43.6\%), which persisted in $13.1 \%$ of cases at 1 month. A fibrinoid anterior chamber reaction was observed

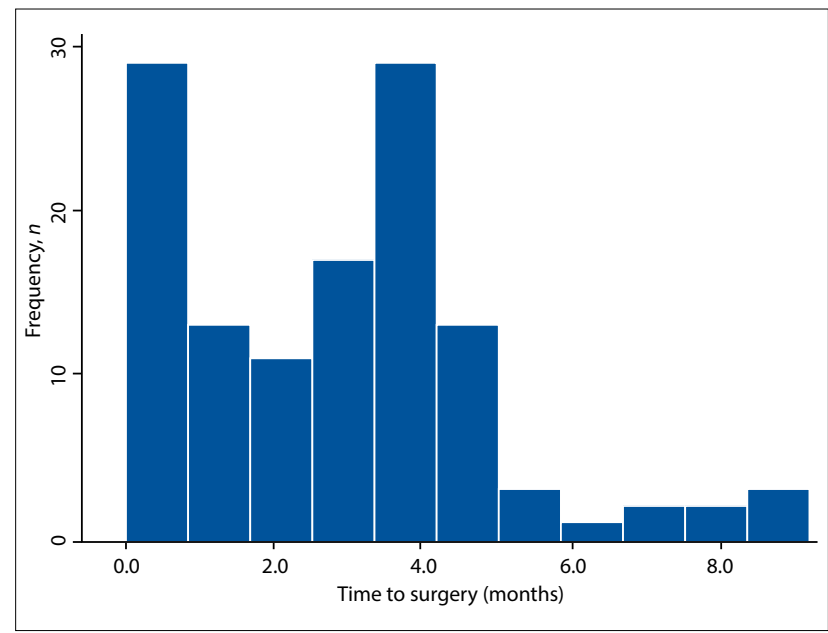

Fig. 1. Waiting time for surgery.

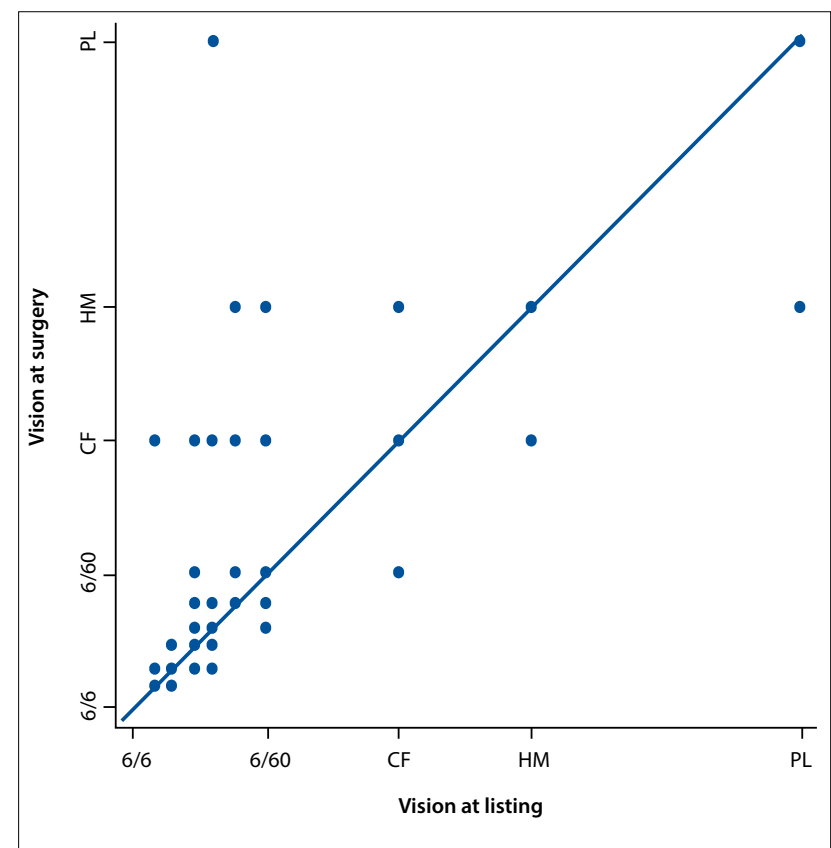

Fig. 2. Change in visual acuity while awaiting surgery (logarithmic scale). (CF = count fingers; $H M=$ hand motions; $P L=$ perception of light.)

in $20.9 \%$ of cases in which cataract surgery was done at the same time as vitrectomy, compared with only $5.3 \%$ of those without concurrent cataract surgery $(p=0.02)$. In $8.0 \%$ of cases reoperation was required for residual subretinal fluid or postoperative vitreous haemorrhage. Anatomical success, defined as an attached retina without tamponade and non-progressive diabetic retinopathy, was achieved in $77.1 \%$ of cases.

Information on visual acuity was available for 113 cases $(91.1 \%)$ at 3 months and for $93(75.0 \%)$ at 6 months. The mean LogMAR acuity was 1.01 (Snellen 6/60) at 3 months and 0.85 (Snellen 6/40) at 6 months, demonstrating a statistically significant improvement $(p=0.01)$. Visual acuity was improved (by at least 0.3 LogMAR units, $n=51$ ) in $54.9 \%$, unchanged (within 0.3 LogMAR units, $n=28$ ) in $30.1 \%$ and worse (by at least 0.3 LogMAR units, $n=13$ ) in $14.0 \%$ of cases at 6 months. Four eyes had no perception of light at 6 months secondary to neovascular glaucoma (Fig. 3).

In our study, the odds of improved vision at 6 months was statistically related to worse visual acuity at the time of surgery (odds ratio (OR) 
2.15; $p=0.048)$. Older age at surgery was associated with a reduced likelihood of visual improvement at 6 months (OR 0.92; $p=0.042$ ). Poor vision in the other eye (counting fingers or worse) did not increase the risk of poor visual outcome $(p=0.58)$. Avoiding iatrogenic retinal breaks (OR $0.13 ; p=0.01$ ) was the most important intraoperative factor associated with better visual outcomes. Visual outcomes were best in the eyes that did not require tamponade and worst in those still containing silicone oil at the 6-month acuity evaluation (Table 3).

\section{Discussion}

The prevalence of type 2 diabetes in SA increased from $5.5 \%$ in 2000 to $9 \%$ in $2009 .{ }^{[4]}$ Current estimates are that $9.7 \%$ of the population older than 30 years are diabetic ${ }^{[2]}$ and that diabetes causes 8000 new cases of blindness annually. ${ }^{[4]}$ Good systemic control of blood glucose, hypertension and other risk factors (primary prevention) and early detection and management of diabetic retinopathy (secondary prevention) are well-established strategies in the battle against diabetic blindness. Digital fundus photography screening is established in many developed countries, and Khan et al. ${ }^{[2]}$ have suggested that this is cost-effective in SA. We are pleased to note the recent efforts of Cook and others to highlight and promote screening

Table 3. Mean visual acuity at 6 months and tamponading agent

\begin{tabular}{llll}
\hline Tamponading agent & $\boldsymbol{n}$ & $\begin{array}{l}\text { Mean LogMAR } \\
\text { acuity }\end{array}$ & $\begin{array}{l}\text { Snellen } \\
\text { equivalent }\end{array}$ \\
\hline None & 35 & 0.70 & $6 / 30$ \\
Air & 6 & 1.03 & $6 / 60$ \\
SF6 gas & 20 & 1.11 & $6 / 60$ \\
C3F8 gas & 7 & 1.08 & $6 / 60$ \\
Silicone oil & & & \\
$\quad$ Oil removed & 12 & 0.73 & $6 / 30$ \\
$\quad$ Oil not yet removed & 13 & 2.03 & CF \\
& & &
\end{tabular}

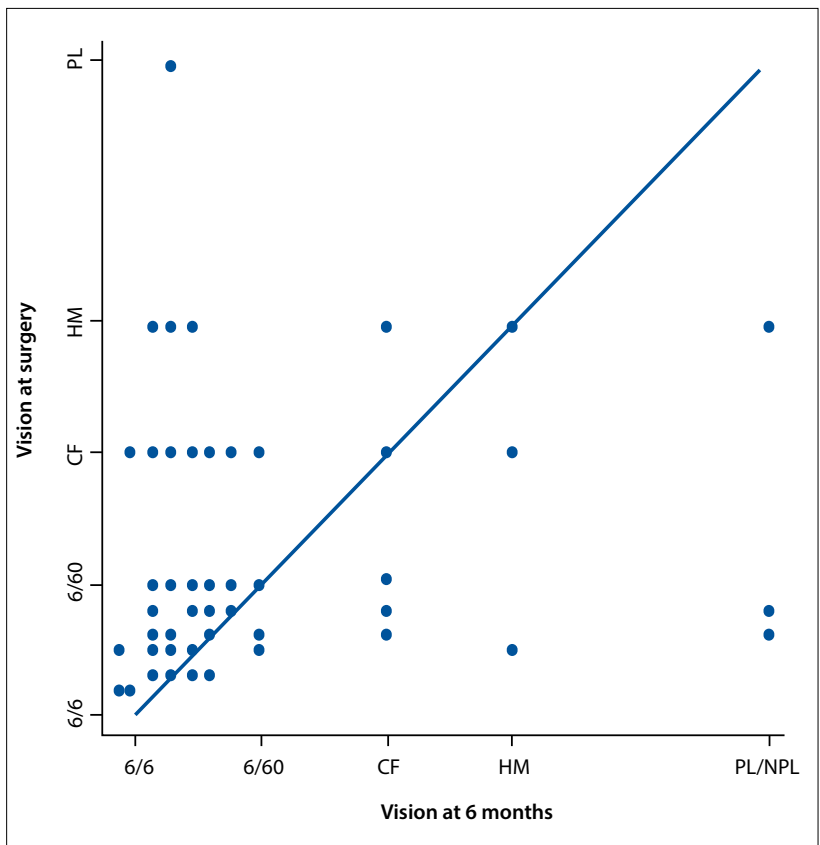

Fig. 3. Change in visual acuity from surgery to 6 months (logarithmic scale). $(C F=$ count fingers; HM = hand motions; $P L / N P L=$ perception of light $/$ no perception of light.) in $S A,{ }^{[5,6]}$ including quality assurance assessment of the graders of fundus photographs. ${ }^{[7]}$ Unfortunately there are currently virtually no established screening programmes, and patients' systemic control is often poor. ${ }^{[8]}$ In addition, the black African population tends to have more aggressive complications of diabetic retinopathy. ${ }^{[9]}$ As a result, patients are referred to tertiary institutions with advanced levels of pathology requiring surgical intervention.

The most common indication for retinal surgery at GSH is the advanced ocular complications of diabetes. Of the patients listed for surgery over our study period, $23.4 \%$ were blind in both eyes (counting fingers or worse) and $20.2 \%$ had one eye assessed as inoperable at presentation. In addition, the progressive nature of this disease resulted in increased morbidity while patients waited for surgery, with $26.2 \%$ of patients suffering further visual loss during this period.

In our hands, retinal surgery was able to achieve anatomical success in $77.1 \%$ of cases and visual stability or improvement in $85.0 \%$, which compares favourably with other series. ${ }^{[10,11]}$ Overall visual acuity improved from a mean of $2 / 60$ preoperatively to $6 / 60$ at 3 months and $6 / 40$ at 6 months. Seventy-four per cent were graded as $6 / 60$ or better at 6 months, which is similar to a series of 174 cases by Yorston et al. ${ }^{[10]}$ Thirty-three per cent achieved 6/12 or better, which compares favourably with a more recent series from the UK. ${ }^{[11]}$ While Snellen visual acuity is limited in its description of visual function, surgery usually achieves stability in an otherwise progressive condition. Patients whose vision remains unchanged after surgery therefore benefit from the reduced risk of declining visual function with time. Our results support vitrectomy as an efficient use of resources. Of the 93 cases with 6 months' follow-up, the number of blind eyes (counting fingers or worse) fell from 41 to 24 , indicating that approximately one in five vitrectomies reverses blindness. Yorston et al.$^{[10]}$ published a similar figure of one in seven procedures.

The time between listing for surgery and the operation has been found to be an important negative predictor of visual outcomes. ${ }^{[1]}$ This is a particularly important factor in resource-limited settings. In previous years, the waiting time in our department was longer than 9 months. With additional theatre time and selection of cases, we reduced the waiting time to an average of 2.9 months (range 1 day - 9 months) at the time of the study. Visual function declined in $26.2 \%$ of our patients during this period. It is likely that waiting times to surgery will continue to be under pressure given the rising prevalence of diabetes and limited secondary prevention strategies in place in SA. The progressive nature of diabetic retinopathy means that prolonged waiting times are also likely to result in more inoperable eyes. In addition, delays in removing silicone oil result in higher rates of oil-related complications such as raised intraocular pressure.

It is an unfortunate reality that criteria for surgery are often changed in order to cope with rising patient numbers. Eyes with a more guarded prognosis may not be offered surgery, or surgery may only be performed on the eye with the better prognosis. The decision whether or not to perform surgery should be based on the best available evidence and balanced with available resources. A number of researchers have attempted to identify preoperative features associated with poor outcome that may be of value in resource-limited environments. Apart from delayed surgery, other factors associated with poorer outcome include older age at surgery, ${ }^{[12]}$ poor preoperative visual acuity, ${ }^{[10,12]}$ poor vision in the other eye at presentation, ${ }^{[10]}$ iris new vessels, ${ }^{[12]}$ poor clinic attendance ${ }^{[11]}$ and retinal detachment involving the macula ${ }^{[10]}$ particularly if longstanding.${ }^{[12]}$ Extensive vitreoretinal adhesions are also associated with poorer outcomes, ${ }^{[10]}$ but this finding is sometimes only determined intraoperatively, making it less useful in patient selection for surgery. Unfortunately, many of our patients present for the first time with many of these poor prognostic features, 
making selection of those likely to benefit from surgery more difficult. It is helpful to note that systemic factors such as hypertension, previous stroke, renal failure and a history of foot ulceration or amputation ${ }^{[10,12]}$ should not be criteria for exclusion unless they pose a high anaesthetic risk or current risk of infection.

We currently offer surgery for one eye if the patient presents with bilateral poor prognostic features. We use the presence of a relative afferent pupil defect and ability to identify correctly the position of a light source (projection) as important clinical criteria for selection in patients with perception of hand motions or light vision only. Many patients presenting with unilateral poor-prognosis eyes will only be offered surgery for the eye with the better prognosis. Our goal is to benefit the maximum number of patients with our current resources. It is important to note that some patients with poor prognostic features do well, ${ }^{[10]}$ as was the case in our series. This puts additional pressure on the clinician to perform surgery, which is not always possible in resource-limited settings.

\section{Study limitations}

Our study has a number of limitations. It was a retrospective analysis that relied on accurate data entry in case notes. Acuity data at 6 months were only available for $75 \%$ of cases, and best visual acuity was based on pinhole testing, as few patients receive refractive correction at our hospital. The degree of macular traction was not specified and would have ranged from minimal traction to complete detachment of the macula, which would have influenced prognosis significantly. In addition, we did not grade the degree of cataract and assume that visually significant cataracts were operated on. We also did not grade the degree of previous laser treatment or vitreoretinal attachments, as suggested by Yorston et al. ${ }^{[10]}$ who found the latter to be an important prognostic factor. Despite these limitations we were encouraged by the overall success of surgery in our hands.

Preoperative bevacizumab (Avastin) was not used during the study period. It has been shown in large studies to reduce surgery time, reduce the need for endocautery and decrease the number of iatrogenic breaks. ${ }^{[13]}$ We now regularly use bevacizumab (off-label) a few days before surgery in eyes with active neovascularisation, which may further improve our outcomes. In addition, the introduction of smaller-gauge (23- and 25-gauge) instruments and bimanual techniques may be associated with fewer posterior iatrogenic breaks. ${ }^{[14]}$ We plan further analysis in our hospital as these techniques and technologies are adopted.

\section{Conclusion}

This study represents the current retinal surgery practice at our centre. The results are in keeping with other publications, and are encouraging given our patients' advanced level of pathology at presentation. They provide useful information for patients regarding surgical outcomes. We recognise the need to keep waiting times for surgery as short as possible, which is likely to become more difficult given the diabetes epidemic. Improvement in primary and secondary preventive strategies is essential in SA and will reduce the number of patients presenting with advanced diabetic eye disease.

\section{References}

1. Read O, Cook C. Retinopathy in diabetic patients evaluated at a primary care clinic in Cape Town. S Afr Med J 2007;97(10):941-942,944

2. Khan T, Bertram MY, Jina R, Mash B, Levitt N, Hofman K. Preventing diabetes blindness: Cost effectiveness of a screening programme using digital non-mydriatic fundus photography for diabetic retinopathy in a primary health care setting in South Africa. Diabetes Res Clin Pract 2013;101(2):170176. [http://dx.doi.org/10.1016/j.diabres.2013.05.006]

3. Mash B, Powell D, du Plessis F, van Vuuren U, Michalowska M, Levitt N. Screening for diabetic retinopathy in primary care with a mobile fundal camera - evaluation of a South African pilot project. S Afr Med I 2007;97(12):1284-1288.

4. Bertram MY Jaswal AVS, van Wyk VP, Levitt NS, Hofman KI. The non-fatal disease burden caused by type 2 diabetes in South Africa, 2009. Glob Health Action 2013;6:19244. [http://dx.doi.org/10.3402/gha.v6io.19244] diabetes in South Africa, 2009. Glob Health Action 2013;6:19244. [http://dx.doi.org/10.3402/gha.v6i0.19244]
. Cook S. Diabetic retinopathy - the Ophthalmology Society of Southern Africa screening programme. Cook S. Diabetic retinopathy - the Ophthalmology Society of Southern A
S Afr Med J 2013;103(7):449-451. [http://dx.doi.org/10.7196/SAMJ.7136]

S Afr Med J 2013;103(7):449-451. [http://dx.doi.org/10.7196/SAMJ.7136]
6. Hofman KJ, Cook C, Levitt N. Preventing diabetic blindness: A priority for South Africa. S Afr Med J 2014;104(10):661-662. [http://dx.doi.org/10.7196/SAMJ.8580]

7. Cook S, Staff RT, Goatman KA, Olson JA, Scottish Diabetic Retinopathy Screening collaborative. Quality assurance in diabetic retinal screening in South Africa. S Afr Med J 2014;104(10):700-704. [http://dx.doi.org/10.7196/SAM].8678]

8. Webb EM, Rheeder P, van Zyl DG. Diabetes care and complications in primary care in the Tshwane distric of South Africa. Prim Care Diabetes 2015;9(2):147-154. [http://dx.doi.org/10.1016/j.pcd.2014.05.002]

9. Thomas RL, Distiller L, Luzio SD, et al. Ethnic differences in the prevalence of diabetic retinopathy in persons with diabetes when first presenting at a diabetes clinic in South Africa. Diabetes Care 2013;36(2):336-341. [http://dx.doi.org/10.2337/dc12-0683]

10. Yorston D, Wickham L, Benson S, Bunce C, Sheard R, Charteris D. Predictive clinical features and Yorston D, Wickham L, Benson S, Bunce C, Sheard R, Charteris D. Predictive clinical features and
outcomes of vitrectomy for proliferative diabetic retinopathy. Br J Ophthalmol 2008:92(3):365-368. outcomes of vitrectomy for proliferative

11. Gupta B, Sivaprasad S, Wong R, et al. Visual and anatomical outcomes following vitrectomy for complications of diabetic retinopathy: The DRIVE UK study. Eye (Lond) 2012;26(4):510-516. [http:// dx.doi.org/10.1038/eye.2011.321]

12. La Heij EC, Tecim S, Kessels AGH, Liem ATA, Japing WJ, Hendrikse F. Clinical variables an their relation to visual outcome after vitrectomy in eyes with diabetic retinal traction detachment. Graefes Arch Clin Exp Ophthalmol 2004;242(3):210-217. [http://dx.doi.org/10.1007/s00417-0030815-5]

13. Zhang Z-H, Liu H-Y, Hernandez-Da Mota SE, et al. Vitrectomy with or without preoperative intravitrea bevacizumab for proliferative diabetic retinopathy: A meta-analysis of randomized controlled trials. Am J Ophthalmol 2013;156(1):106.e2-115.e2. [http://dx.doi.org/10.1016/j.ajo.2013.02.008]

14. Gosse E, Newsom R, Lochhead J. The incidence and distribution of iatrogenic retinal tears in 20-gauge and 23-gauge vitrectomy. Eye (Lond) 2012;26(1):140-143. http://dx.doi.org/10.1038/eye.2011.289] 